Research Paper

\title{
Circulating microRNA Panel as a Novel Biomarker to Diagnose Bisphosphonate-Related Osteonecrosis of the Jaw
}

\author{
Rui Yang ${ }^{1 *}$, Yurong Tao ${ }^{2 *}$, Chao Wang ${ }^{1}$, Yi Shuai ${ }^{3 凶}$, Lei Jin ${ }^{3 凶}$ \\ 1. Department of Stomatology, PLA Army General Hospital, Beijing, 100000, People's Republic of China; \\ 2. Department of Gastroenterology, PLA Army General Hospital, Beijing, 100000, People's Republic of China; \\ 3. Department of Stomatology, Nanjing General Hospital of Nanjing Military Command, Nanjing, Jiangsu 210002, People's Republic of China. \\ *These authors contributed equally to the study.
}

$\square$ Corresponding authors: Lei Jin, MD. PhD. Tel: +86-25-80861166, Fax: +86-25-80863661, E-mail: Ljin@nju.edu.cn; Yi Shuai, MD. PhD. Tel: +86-25-80861166, Fax: +86-25-80863661, E-mail: handsy@126.com.

(c) Ivyspring International Publisher. This is an open access article distributed under the terms of the Creative Commons Attribution (CC BY-NC) license (https://creativecommons.org/licenses/by-nc/4.0/). See http://ivyspring.com/terms for full terms and conditions.

Received: 2018.05.31; Accepted: 2018.11.02; Published: 2018.11.22

\begin{abstract}
There is no defined biomarker for BRONJ diagnosis with satisfactory performance in clinic. In this study, we established the BRONJ model and selected 7 microRNAs as candidate for BRONJ diagnosis from microRNA microarray reported by other research. Dysregulated microRNAs during BRONJ were detected and validated in two independent animal experiments using serum samples. In the first part, serum miR-21, miR-23a and miR-145 were significantly altered in between BRONJ and control group. And an Indice was constructed as $-0.032+(0.154 \times$ miR-21 $)+(0.145 \times$ miR-23a $)+$ $(-0.700 \times$ miR-145) using logistic regression model to improve diagnostic performance. The performance of Indice to differentiate BRONJ subjects from control group was analyzed as AUC of $0.82(95 \% \mathrm{Cl}$, $0.72-0.92)$ or $0.85(95 \% \mathrm{Cl}, 0.73-0.97)$ in the first or second part. Moreover, the predictive performance of Indice to discriminate BRONJ-1 $\mathrm{w}$ and BRONJ-4w from control group was displayed as AUC of 0.65 $(95 \% \mathrm{Cl}, 0.47-0.84)$ or $0.75(95 \% \mathrm{Cl}, 0.60-0.91)$, which was better than individual circulating microRNAs. In addition, the expressions of candidate microRNAs were validated in human samples. Consequently, we investigated a combined Indice constructed with circulating microRNAs for BRONJ diagnosis and prediction.
\end{abstract}

Key words: bisphosphonate-related osteonecrosis of the jaw, circulating microRNA, biomarker, diagnosis

\section{Introduction}

Bisphosphonates are commonly known as powerful inhibitors of osteoclastogenesis, which have been used to prevent the osteoporotic bone loss and reduce the risk of osteoporotic fracture in patients suffered from postmenopausal osteoporosis[1].

Although bisphosphonates markedly ameliorate osteoporosis, their side-effects largely limit the clinical application of these drugs for osteoporosis treatment. Bisphosphonate-related osteonecrosis of the jaw (BRONJ) has been recognized as a rare but severe adverse event associated with bisphosphonates administration[2]. It has been reported that oral and maxillofacial surgery may obviously increase the risk of such a drug-related complication, which mainly attributes to impaired oral wound healing[3]. In addition, the risk of BRONJ is positive related with the dose and accumulation of bisphosphonates exposure[4].

BRONJ has been reported for about fifteen years. However, the exact mechanism of this drug-related disease seems to be multi-factorial and remains elusive, resulting in management failure of BRONJ. Apart from age, sex, smoking, oral hygiene, infection and systemic diseases, genetic background has been frequently reported to be a predisposing element for initiation of osteonecrosis of the jaw $(\mathrm{ONJ})[5,6]$. Emerging evidences showed genetic association of diverse genes dysregulation with BRONJ[7-9], 
suggesting that altered gene expressions might be potential biomarkers for BRONJ diagnosis. In addition, since BRONJ is closely related with bone metabolic disorders, bone turnover markers have been emerging to support diagnosis of BRONJ[10-13]. Nevertheless, inconsistent diagnostic performances were observed in various researches and no approved clinical guide has been established to manage BRONJ. On account of the increasing usage of anti-resorptive pharmaceuticals like bisphosphonates for various bone disorders, it is essential to research and develop specific and stable biomarkers to identify subjects at high risk of developing BRONJ.

Recently, a novel approach has been proposed to diagnose diseases using circulating microRNAs, which is a type of microRNAs with specificity and stability existing in body fluids[14]. The diagnostic performances of circulating microRNAs have been validated in numerous diseases, including cancers [15], heart diseases[16], osteoporosis[17], etc. However, no research has been reported to diagnose BRONJ using circulating microRNAs. A recent research described an altered microRNA expression profile in multiple myeloma patients with BRONJ, suggesting that post-transcriptional regulation might be crucial for BRONJ development[18]. They obtained total RNAs of circulating lymphocytes for microRNA analysis from healthy subjects and multiple myeloma patients with BRONJ. A class of fourteen microRNAs markedly elevated in patients with BRONJ, including miR-16-1, miR-21， miR-23a, miR-28, miR-101-1, miR-124-1, miR-129-1, miR-139, miR-145, miR-149, miR-202, miR-221, miR-424 and miR-520[18]. Most of aforementioned microRNAs have been revealed to regulate bone metabolism and influence bone remodeling, indicating that microRNA signatures might exert their specific regulation on osteoblastogenesis and osteoclastogenesis in BRONJ. Circulating microRNAs are closely related to tissue or cell specific microRNAs, thus the altered microRNAs profile might be a promising resource for circulating microRNA biomarkers study.

In this study, we investigated three discriminative circulating microRNAs and a combined microRNAs panel based on the data from microRNA microarray of Caterina Musolino's study[18] to propose a novel strategy for diagnosing BRONJ and alert its initiation.

\section{Materials and Methods}

\section{Ethics}

All animals were purchased from Beijing Vital River Laboratory Animal Technology Co., Ltd. All the animal study protocols were approved by the Animal Care Committee of the PLA Army General Hospital, Beijing, China, which was on the basis of NIH Guide for the Care and Use of Laboratory Animals. The collection and usage of human sera were approved by the Institutional Review Board for Human Subjects Research of PLA Army General Hospital (Ethic NO. 2018-50). All the participants were provided written informed consents for their donation of sera in this research.

\section{Animal groups and model establishment}

A total of 140 female Sprague-Dawley rats (10-12 months old, 240-280 g) were involved in this study. All the rats were separated into two parts, 60 rats were used for the first part, while the rest was used for the second part. In the first part, 60 rats were equally divided into control group and BRONJ group, while 80 rats were equally divided into four groups in the second part, namely control group, BRONJ-1w group, BRONJ-4w group and BRONJ-8w group. The model of BRONJ was established according to the protocols reported by $R$. Nicole Howie and his colleagues [19]. Briefly, rats in BRONJ group were weekly administrated with zoledronate (LifeSciences, USA) at a dose of $80 \mu \mathrm{g} / \mathrm{kg}$ body weight (iv.) for 13 weeks, which was followed by first and second molars extraction on the left side. Meanwhile, rats in control group were intravenously injected with phosphate-buffered saline. All the operations were conducted under anesthesia. All the rats were housed under specific pathogen-free conditions with a temperature of $24^{\circ} \mathrm{C}$, cycles of $12 \mathrm{~h}$ light/12 h dark, and humidity of $50 \%-55 \%$. The jawbones were obtained for microCT analysis. After microCT scanning, jawbones were decalcified, and then for H\&E staining and TRAP staining (Sigma-Aldrich) according to the protocol introduction.

\section{Whole blood collection and serum preparation}

For rats sera, all the rats were fasted half day before blood collection. $5 \mathrm{~mL}$ venous blood was collected from rats' abdominal vein under anesthesia in the morning. For human sera, fasting blood samples $(5 \mathrm{~mL})$ were collected via ulnar vein puncture and imported into the sterile vacuous dry tube without any anticoagulation agents, in the morning (8:00 am to 12:00 am). Eleven control participants and six BRONJ patients were recruited (Table S1). The blood samples were stored for 30 minutes at room temperature, then were centrifuged at $4^{\circ} \mathrm{C}, 1000 \times \mathrm{g}$, for $15 \mathrm{~min}$ to permit the completely dissociation of cell and cell debris free serum. 


\section{Circulating microRNAs isolation, cDNA preparation and q-RT-PCR analysis}

Total RNA was isolated from $200 \mu \mathrm{L}$ serum using mirVana Paris Kit (Ambion, USA) following the provided protocol. A spike-in reference of Lyophilized C.elegans miR-39 miRNA mimic (Qiagen, Germany) was used to normalize the data according to the manufacturer's instruction. The microRNAs were reversed to cDNA using miScript II RT Kit (Qiagen, Germany).

Q-RT-PCR analysis of microRNAs was conducted using miScript SYBR Green PCR Kit (Qiagen, Germany) with a 7500 Real-Time PCR System (LifeSciences, USA) according to the manufacturer's protocol. The reaction procedure was set as follows: Step 1: $95^{\circ} \mathrm{C} 30 \mathrm{~s}$; Step 2: PCR reaction, GO TO: 39 (40 cycles), $95^{\circ} \mathrm{C} 5 \mathrm{~s}, 60^{\circ} \mathrm{C} 30 \mathrm{~s}$; Step 3: Melt Curve. Relative expressions of candidate microRNAs were normalized by the level of C.elegans miR-39 miRNA mimic using 2- $-\Delta c t$ method. Forward primers sequences for candidate microRNAs were displayed in Table S2, and the universal reverse primer was supplied with the kit.

\section{Statistical analysis}

All the data were calculated in SPSS 13.0. All the figures were graphed in GraphPad Prism 7. Student's $t$ test or non-parametric test was used for comparison of control and BRONJ groups. The Kruskal-Wallis test or non-parametric test was used for pairwise comparisons of control, BRONJ-1w, BRONJ-4w and BRONJ-8w groups. The combined Indice was developed using a logistic regression model based on the data from the first part. Receiver operating characteristic (ROC) curve and area under ROC curve (AUC) were used for the diagnostic performance exhibition of each microRNA and Indice. The cutoff points were defined according to the designed sensitivity of $80.00 \%$. The threshold value for statistical significance was set at $p<0.05$.

\section{Results}

\section{Establishment of the BRONJ model}

The jaws of Control and BRONJ group were all scanned by microCT. The data revealed that all the rats in the BRONJ group developed necrosis of the jaw. Severe lesions were observed in the jaws of BRONJ group, whereas the Control group showed a complete wound healing (Fig. S1A, B). Furthermore, the HE staining confirmed the data of microCT scanning. Bone lesions and inflammatory infiltration were observed in the sections of BRONJ group, however the Control group showed healthy structure (Fig. S1C, D). In addition, much fewer TRAP positive areas were observed in BRONJ group compared to Control group (Fig. S1E, F). The aforementioned results suggested that the BRONJ model were successfully established.

\section{Selection of candidate circulating miRNAs (first part)}

Seven microRNAs (miR-21, miR-23a, miR-28, miR-124-1, miR-129-1, miR-145 and miR-149) from Caterina Musolino' study were selected to analyze candidate circulating microRNAs in BRONJ, according to the criteria of high preservation and correlation with bone metabolism. Among the candidate microRNAs, miR-21 and miR-23a were increased in serum of BRONJ compared to control group, while miR-145 was decreased. Whereas, miR-28, miR-129-1 and miR-149 showed no difference in serum between BRONJ and control group, and miR-124-1 could not detected in serum. The three altered microRNAs (miR-21, miR-23a and miR-145) were then analyzed using ROC curve system. However, miR-21, miR-23a and miR-145 presented a moderate diagnostic performance with the respective AUC value of 0.70 (95\% CI, $0.57-0.84), 0.72$ (95\% CI, $0.59-0.85)$ or 0.65 (95\% CI, 0.51-0.79). The diagnostic information was displayed in Figure 1, 2 and Table 1. In addition, miR-21, miR-23a and miR-145 were also detected in the human derived sera to validate the performance. Similar to aforementioned results, miR-21 and miR-23a were elevated in BRONJ compared to control group, while miR-145 was declined (Figure S2).

\section{Diagnostic performance of combined Indice in BRONJ diagnosis (first and second part)}

The combined Indice was developed as $-0.032+(0.154 \times$ miR-21 $)+(0.145 \times$ miR-23a $)+(-0.700 \times$ miR-145) according to a logistical regression model (first part). The AUC of Indice was $0.82(95 \% \mathrm{CI}$, 0.72-0.92) to differentiate BRONJ from control group, which was highly improved compared with individual microRNA, as well as the diagnostic specificity $(63.33 \%)$ and accuracy $(71.67 \%)$ based on a designed sensitivity of $80.00 \%$. The diagnostic information was presented in Figure 1, 2 and Table 1.

Another independent experiment (second part) was conducted to validate the diagnostic performance of candidate microRNAs and combined Indice in BRONJ diagnosis. In accordance with the first part, the AUC (0.85), specificity $(70.00 \%)$ and accuracy $(75.00 \%)$ of combined Indice also outclassed those of miR-21, miR-23a and miR-145, suggesting a better diagnostic performance of combined Indice compared with individual microRNAs. The diagnostic information was presented in Figure 3 and Table 2. 
A
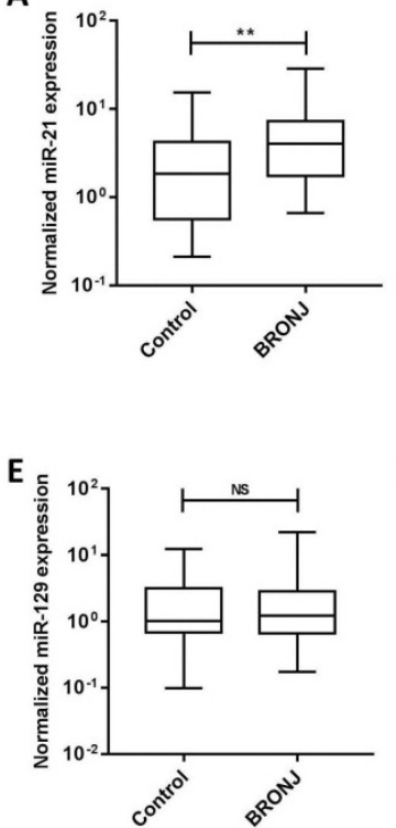

B

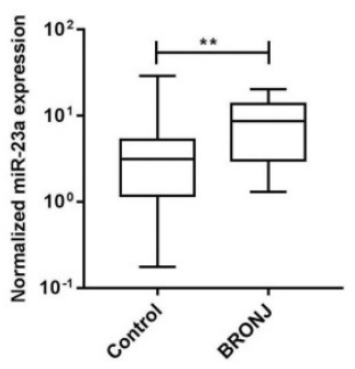

$\mathbf{F}$

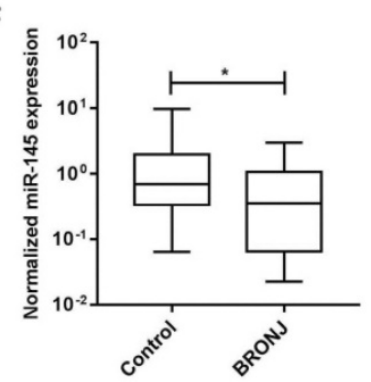

C

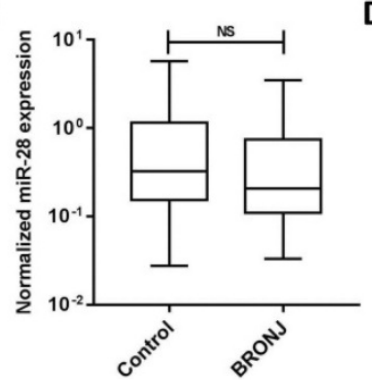

G

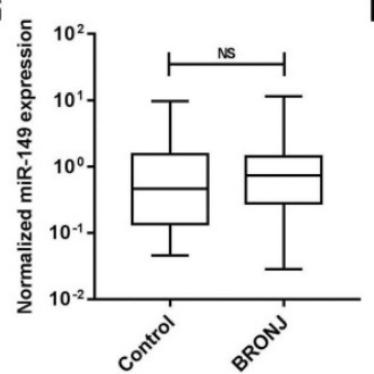

D

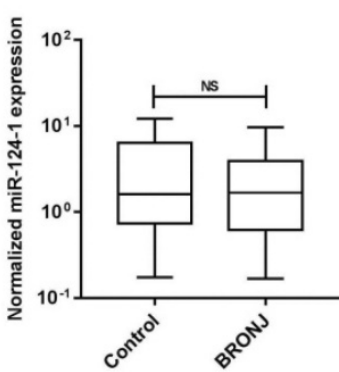

H

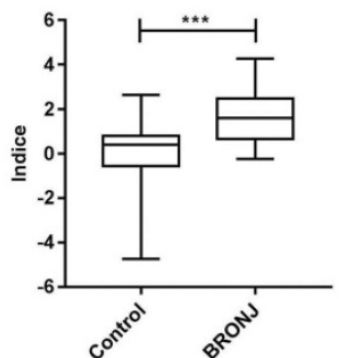

Figure 1. The normalized expressions of rno-miR-21, rno-miR-23a, rno-miR-28, rno-miR-124-1, rno-miR-129-1, rno-miR-145, rno-miR-149 and combined Indice in the sera of the control and BRONJ groups (first part) $\left({ }^{*} \mathrm{p}<0.05,{ }^{* *} \mathrm{p}<0.01,{ }^{* * * *} \mathrm{p}<0.001\right.$, NS: no significance).

A

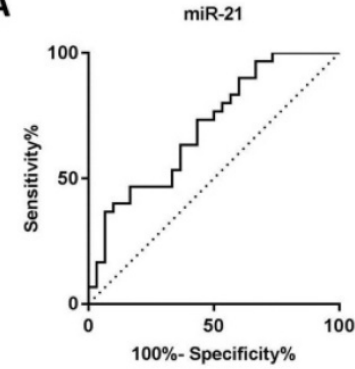

E

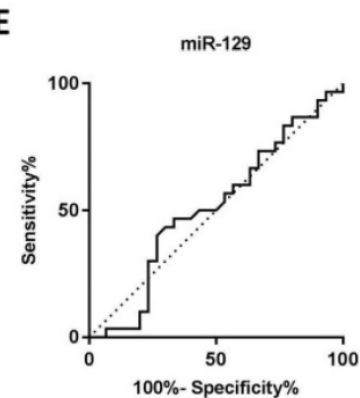

B

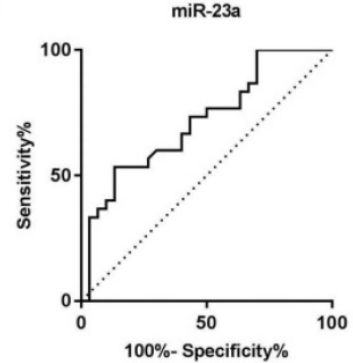

$\mathbf{F}$

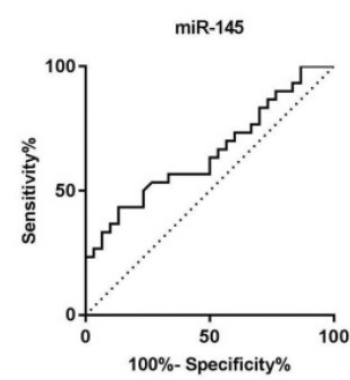

C

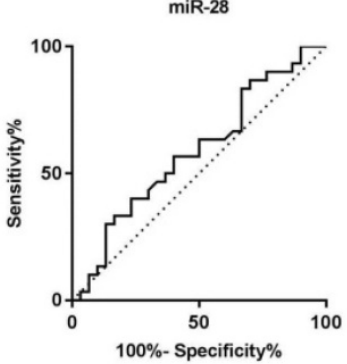

G

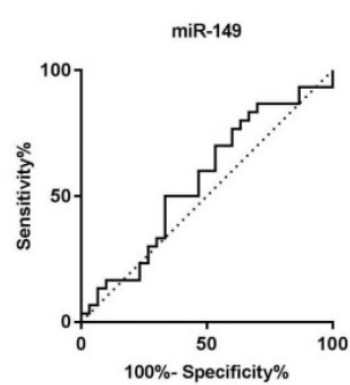

D

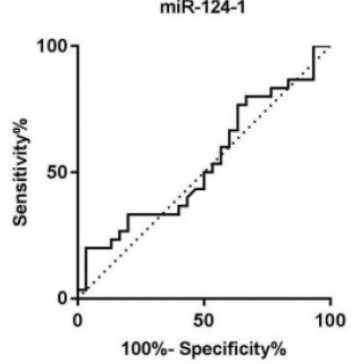

H

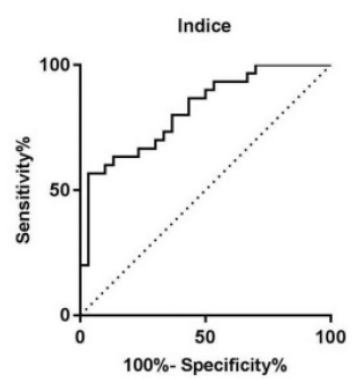

Figure 2. ROC curves of rno-miR-21, rno-miR-23a, rno-miR-28, rno-miR-124-1, rno-miR-129-1, rno-miR-145, rno-miR-149 and combined Indice in the sera of the control and BRONJ groups (first part).

Table 1. Diagnostic performance of individual microRNA and Indice on BRONJ (first part)

\begin{tabular}{|c|c|c|c|c|c|c|c|c|c|c|}
\hline miRNA & $\begin{array}{l}\text { Cutoff } \\
\text { value }\end{array}$ & $\begin{array}{l}\text { AUC } \\
(95 \% \mathrm{CI})\end{array}$ & $p$ value & $\begin{array}{l}\text { Designed } \\
\text { Sensitivity\# }(\%)\end{array}$ & Specificity (\%) & Accuracy (\%) & $\begin{array}{l}\text { True } \\
\text { positive }\end{array}$ & $\begin{array}{l}\text { True } \\
\text { negative }\end{array}$ & $\begin{array}{l}\text { False } \\
\text { positive }\end{array}$ & $\begin{array}{l}\text { False } \\
\text { negative }\end{array}$ \\
\hline miR-21 & 1.42 & $0.70(0.57-0.84)$ & 0.007 & 80.00 & 46.67 & 63.34 & 24 & 14 & 16 & 6 \\
\hline $\operatorname{miR}-23 a$ & 2.23 & $0.72(0.59-0.85)$ & 0.004 & 80.00 & 36.67 & 58.34 & 24 & 11 & 19 & 6 \\
\hline miR-145 & 0.85 & $0.65(0.51-0.79)$ & 0.041 & 80.00 & 33.33 & 56.67 & 24 & 10 & 20 & 6 \\
\hline Indice & 0.64 & $0.82(0.72-0.92)$ & $<0.001$ & 80.00 & 63.33 & 71.67 & 24 & 19 & 11 & 6 \\
\hline
\end{tabular}

Note: Designed sensitivity\#: the performance was considered by defining a cutoff value corresponding to fixing the sensitivity to $80.00 \%$. 
A

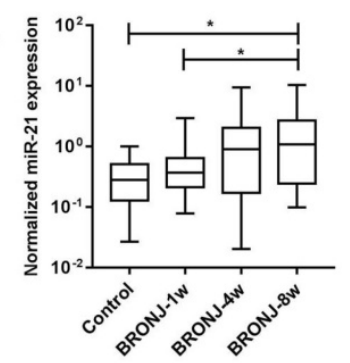

E
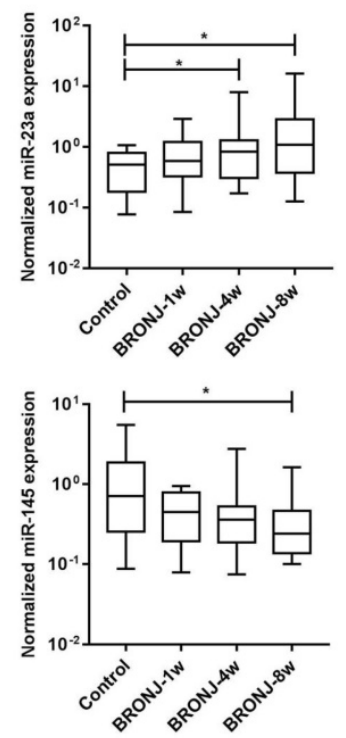

$\mathbf{M}$

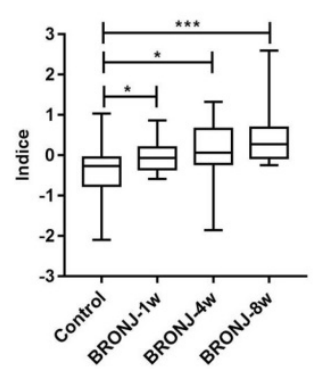

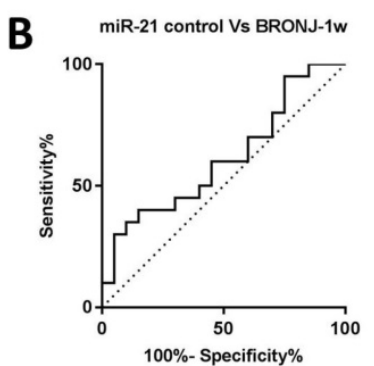

$\mathbf{F}$

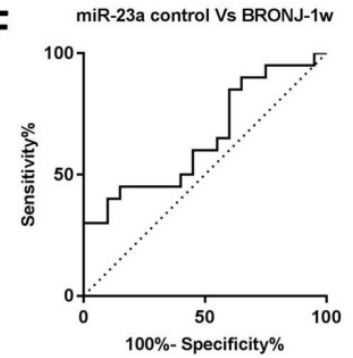

J miR-145 control Vs BRONJ-1w

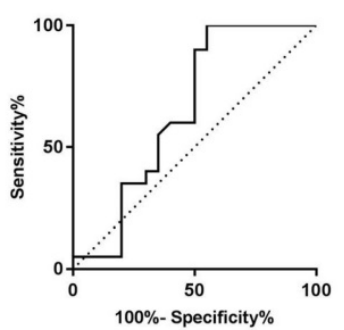

$\mathbf{N}$

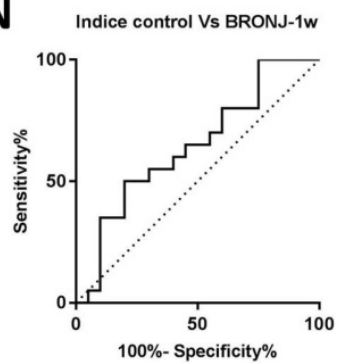

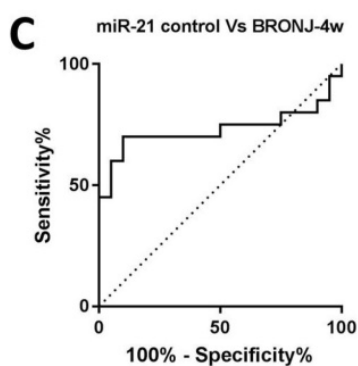

$\mathbf{G}$

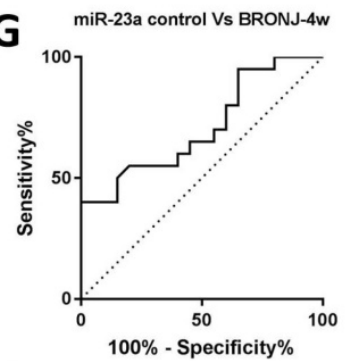

K miR-145 control Vs BRONJ-4w

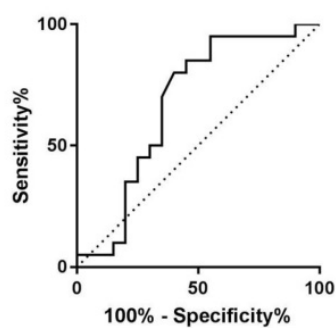

O Indice control Vs BRONJ-Aw

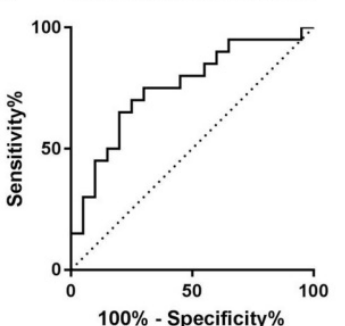

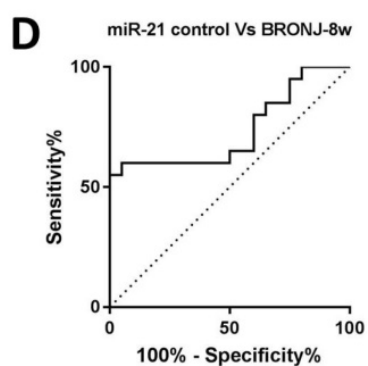

H miR-23a control Vs BRONJ-8w

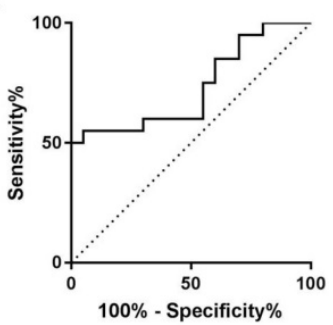

$\mathbf{L}$

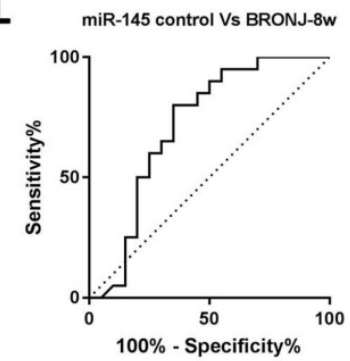

$\mathbf{P}$

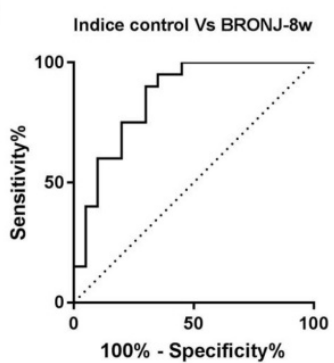

Figure 3. The normalized expressions and ROC curves of rno-miR-21, rno-miR-23a, rno-miR-145 and combined Indice in the sera of the control, BRONJ-1w, BRONJ-4w and BRONJ-8w groups (second part) $\left({ }^{*} p<0.05,{ }^{* *} p<0.01,{ }^{* * * *} p<0.001\right)$.

Table 2. Diagnostic and predictive performance of individual microRNA and Indice on BRONJ progression (second part)

\begin{tabular}{|c|c|c|c|c|c|c|c|c|c|c|}
\hline miRNA & $\begin{array}{l}\text { Cutoff } \\
\text { value }\end{array}$ & $\begin{array}{l}\text { AUC } \\
(95 \% \mathrm{CI})\end{array}$ & $p$ value & $\begin{array}{l}\text { Designed } \\
\left.\text { Sensitivity }{ }^{\#} \%\right)\end{array}$ & $\begin{array}{l}\text { Specificity } \\
(\%)\end{array}$ & Accuracy (\%) & $\begin{array}{l}\text { True } \\
\text { positive }\end{array}$ & $\begin{array}{l}\text { True } \\
\text { negative }\end{array}$ & $\begin{array}{l}\text { False } \\
\text { positive }\end{array}$ & $\begin{array}{l}\text { False } \\
\text { negative }\end{array}$ \\
\hline \multicolumn{11}{|c|}{ Control Vs BRONJ-1w } \\
\hline miR-21 & 0.21 & $0.61(0.44-0.79)$ & 0.224 & 80.00 & 30.00 & 55.00 & 16 & 6 & 14 & 4 \\
\hline $\operatorname{miR}-23 a$ & 0.32 & $0.64(0.48-0.82)$ & 0.099 & 80.00 & 40.00 & 60.00 & 16 & 8 & 12 & 4 \\
\hline miR-145 & 0.80 & $0.65(0.47-0.83)$ & 0.108 & 80.00 & 50.00 & 65.00 & 16 & 10 & 10 & 4 \\
\hline Indice & -0.37 & $0.65(0.47-0.84)$ & 0.048 & 80.00 & 55.00 & 67.50 & 16 & 11 & 9 & 4 \\
\hline \multicolumn{11}{|c|}{ Control Vs BRONJ-4w } \\
\hline miR-21 & 0.12 & $0.73(0.55-0.91)$ & 0.013 & 80.00 & 25.00 & 52.50 & 16 & 5 & 15 & 4 \\
\hline miR-23a & 0.30 & $0.71(0.55-0.87)$ & 0.024 & 80.00 & 40.00 & 60.00 & 16 & 8 & 12 & 4 \\
\hline miR-145 & 0.53 & $0.67(0.50-0.85)$ & 0.049 & 80.00 & 55.00 & 67.50 & 16 & 11 & 9 & 4 \\
\hline Indice & -0.25 & $0.75(0.60-0.91)$ & 0.006 & 80.00 & 55.00 & 67.50 & 16 & 11 & 9 & 4 \\
\hline \multicolumn{11}{|c|}{ Control Vs BRONJ-8w } \\
\hline miR-21 & 0.24 & $0.74(0.57-0.90)$ & 0.011 & 80.00 & 40.00 & 60.00 & 16 & 8 & 12 & 4 \\
\hline miR-23a & 0.37 & $0.73(0.57-0.89)$ & 0.010 & 80.00 & 40.00 & 60.00 & 16 & 8 & 12 & 4 \\
\hline miR-145 & 0.49 & $0.71(0.54-0.88)$ & 0.021 & 80.00 & 65.00 & 72.50 & 16 & 13 & 7 & 4 \\
\hline Indice & -0.09 & $0.85(0.73-0.97)$ & $<0.001$ & 80.00 & 70.00 & 75.00 & 16 & 14 & 6 & 4 \\
\hline
\end{tabular}

Note: Designed sensitivity": the performance was considered by defining a cutoff value corresponding to fixing the sensitivity to $80.00 \%$. 


\section{Predictive performance of combined Indice in BRONJ progress (second part)}

Apart from diagnosis, prediction of $\mathrm{BRONJ}$ initiation might also significant for BRONJ management. Therefore, this study tried to distinguish different phases of BRONJ development. Only miR-21 could distinguish BRONJ-4w from control group, whereas none of these three microRNAs could distinguish BRONJ-1w from control group. However, the Indice was gradually increased among control, BRONJ-1w, BRONJ-4w and BRONJ-8 group, meanwhile the combined Indice effectively differentiated BRONJ-1w and BRONJ-4w from control group with respective AUC of 0.65 (95\% CI, 0.47-0.94) or 0.75 (95\% CI, 0.60-0.91), indicating that combined Indice might be a potential predictor of BRONJ progress. The predictive information was presented in Figure 3 and Table 2.

\section{Discussion}

Bisphosphonate-related osteonecrosis of the jaw has been reported for more than ten years. However, there is no certain identification of biomarkers for BRONJ diagnosis. In current study, we evaluated seven circulating microRNAs and found three of them (miR-21, miR-23a and miR-145) markedly differing between control and BRONJ groups. Nevertheless, none of the AUC values was greater than 0.80, indicating a moderate diagnostic effect of the three selected microRNAs. Therefore, we further investigated a combined Indice $(-0.032+(0.154 \times$ miR-21 $)+(0.145 \times$ miR-23a $)+(-0.700 \times$ miR-145) $)$ based on a logistic regression model, which highly improved diagnostic efficiency of BRONJ with an AUC of 0.82 (95\% CI, 0.72-0.92) , specificity of $63.33 \%$, and accuracy of $71.67 \%$ compared to individual microRNAs. The expressions of miR-21, miR-23a and miR-145 were detected using human samples, which were consistent with aforementioned observations, suggesting a conserved role of these three microRNAs in BRONJ initiation and development. There were only 11 healthy controls and $6 \mathrm{BRONJ}$ patients being included, the broader variation of miRNA expressions in human samples was partially credit to the individual difference and small sample size. In addition, a validation study was absent for human sample analysis because of the limited sample size. Therefore a larger sample size is needed for further study. Moreover, the diagnostic performance of Indice has been validated using an independent animal experiment, suggesting that the combined Indice is a potent biomarker for BRONJ diagnosis. In addition, although individual microRNA failed to predict BRONJ initiation after 1 week or 4 weeks induction, the Indice effectively distinguished
BRONJ-1w and BRONJ-4w from control subjects. To our knowledge, it is the first time to investigated the circulating microRNA formed Indice to be a promising biomarker for diagnosing or predicting BRONJ initiation and development.

One of the likely etiologies of BRONJ is destructive bone remodeling triggered by an imbalance of bone resorption and bone formation. Therefore, bone turnover markers have been reported to diagnose BRONJ, including C-terminal telopeptide of type I collagen (CTx), N-telopeptide of type I collagen (NTX), tartrateresistant acid phosphatase isoform $5 b$ (TRACP $5 b$ ), receptor activator for nuclear factor-K B ligand (RANKL)/osteoprotegerin (OPG), total alkaline phosphatase (tALP) and bone-specific alkaline phosphatase (BAP)[10-13]. However, their diagnostic performance has not been well defined. According to Jin-Woo Kim' study, only serum TRACP $5 \mathrm{~b}$ showed an AUC of 0.807, whereas other serum markers showed poor performance[12]. Similarly, Antonia Kolokythas and colleagues observed an elevation of salivary NTX in medication-related osteonecrosis of the jaws[13]. Additionally, Vivek Thumbigere-Math and colleagues revealed that none of the reported bone turnover markers differentiate BRONJ from healthy control[11]. Moreover, Vivek Thumbigere-Math's research also investigated a minor augment of angiogenic marker-vascular endothelial growth factor (VEGF) in BRONJ compared to healthy control[11]. However, no comprehensive diagnostic evaluation and no validated experiment were provided in the previous studies. In our system, we systemically investigated diagnostic performance of Index with a high AUC to distinguish BRONJ from control group using two independent experiments.

Prediction and early intervention might be beneficial for BRONJ management. Jin-Woo Kim and colleagues reported a sharp decrease of CTx, RANKL and TRACP $5 \mathrm{~b}$ in BRONJ subjects (6 weeks after BRONJ induction) compared to normal control, suggesting a potential role for alerting BRONJ[12]. However, six weeks after BRONJ induction seems a little late for BRONJ therapy. In our study, we investigated circulating microRNAs constructed Indice to forecast BRONJ initiation only one week after BRONJ induction, which is prospected to be a predictive marker for BRONJ.

Although miR-21 has been reported to be a crucial regulator in bone metabolism, its detailed mechanisms remain complex. According to the researches, miR-21 improved osteogenic differentiation by targeting Smad7, Spry1 and PLAP1[20-22]. Apart from influence on osteblastogenesis, miR-21 also plays a critical role in up-regulation of osteoclastogensis via augmenting RANKL and 
suppressing OPG expression[23]. In addition, miR-21 also displayed positive correlation with particleinduced osteolysis pathogenesis[24], while knocking down of miR-21 resulted in osteoclastogenesis restriction[25]. In our system, circulating miR-21 was up-regulated during BRONJ progress, which was consistent with the miR-21 variation in pro-osteoclastogenesis. MiR-23a has been reported to suppress osteoblastic differentiation by regulating TGF- $\beta$ signaling, Tmem64 or LRP5[26-28]. Furthermore, miR-23a targeting LRP5 was also closely related with steroid-associated femoral head necrosis, meanwhile miR-23a inhibitor ameliorated osteonecrosis in an animal model[29]. MiR-145 not only has been validated to be increased during osteoblast differentiation[30], but also to inhibit monocytes related osteoclastogenesis induced by RANKL[31]. However, silencing of miR-145 resulted in rescued steroidinduced avascular necrosis of the femoral head[32]. In our study, an elevation of serum miR-21, miR-23a, and a reduction of serum miR-145 were observed in BRONJ subjects, which were corresponding to the cellular functions reported by previous researches. However, the correlation of intracellular microRNAs and circulating microRNAs in BRONJ development remains unknown, as well as the detailed mechanisms. Further studies are required to understand the role of these microRNAs in BRONJ development.

However, some limitations of current study should be considered and further modified. Firstly, a high throughput sequencing of serum microRNAs might be preferable to obtain data base and bioinformatics rather than selection from literature review. Secondly, a target gene prediction and gain or loss experiments should be conducted to reveal the detailed mechanisms of dysregulated circulating microRNAs in BRONJ development.

\section{Supplementary Material}

Supplementary figures and tables.

http://www.medsci.org/v15p1694s1.pdf

\section{Abbreviations}

BRONJ: bisphosphonate-related osteonecrosis of the jaw; ONJ: osteonecrosis of the jaw; ROC: receiver operating characteristic; AUC: curve and area under ROC curve; CTx: C-terminal telopeptide of type I collagen; NTX: N-telopeptide of type I collagen; TRACP 5b: tartrateresistant acid phosphatase isoform 5b; RANKL: receptor activator for nuclear factor-K B ligand; OPG: osteoprotegerin; tALP: total alkaline phosphatase; BAP: bone-specific alkaline phosphatase; VEGF: vascular endothelial growth factor.

\section{Acknowledgements}

This work was financially supported by grants from the Jiangsu Province "333" High Level Talents Cultivation Project (Grant No: BK2016544), Jiangsu Province Medical Key Talents Project (Grant No: ZDRCA2016095) and Military Medical Science and Technology Youth Cultivation Project (Grant No: 17QNP054).

We thank Shengqi Zang, Yulin An, Xiaolei Shi, Lei Zhu and Rui Mu for intellectual support of BRONJ related information in Department of Stomatology, Nanjing General Hospital of Nanjing Military Command, Nanjing, Jiangsu, People's Republic of China.

\section{Authorship}

Lei Jin, Yi Shuai, Rui Yang and Yurong Tao conceived and designed the study. Lei Jin and Yi Shuai supervised the project. Rui Yang and Yurong Tao conducted the experiments, analyzed the data and wrote the manuscript. Chao Wang took part in sample collection and provided some technical supports. Lei Jin and Yi Shuai reviewed and revised the manuscript.

\section{Competing Interests}

The authors have declared that no competing interest exists.

\section{References}

1. Cosman F, de Beur SJ, LeBoff MS, Lewiecki EM, Tanner B, Randall S, et al. Clinician's Guide to Prevention and Treatment of Osteoporosis. Osteoporos Int. 2014; 25: 2359-81.

2. Spanou A, Lyritis GP, Chronopoulos E, Tournis S. Management of bisphosphonate-related osteonecrosis of the jaw: a literature review. Oral Dis. 2015; 21: 927-36.

3. Bodem JP, Kargus S, Eckstein S, Saure D, Engel M, Hoffmann J, et al. Incidence of bisphosphonate-related osteonecrosis of the jaw in high-risk patients undergoing surgical tooth extraction. J Craniomaxillofac Surg. 2015; 43: 510-4.

4. Kuroshima S, Sasaki M, Nakajima K, Tamaki S, Hayano H, Sawase T. Prevalence of bisphosphonate-related osteonecrosis of the jaw-like lesions is increased in a chemotherapeutic dose-dependent manner in mice. Bone. 2018; 112: $177-86$

5. Kim JH, Ko YJ, Kim JY, Oh Y, Hwang J, Han S, et al. Genetic investigation of bisphosphonate-related osteonecrosis of jaw (BRONJ) via whole exome sequencing and bioinformatics. PLoS One. 2015; 10: e0118084

6. Kim KY, Zhang X, Cha IH. Identifying a combined biomarker for bisphosphonate-related osteonecrosis of the jaw. Clin Implant Dent Relat Res. 2018; 20: 191-8.

7. Arduino PG, Menegatti E, Scoletta M, Battaglio C, Mozzati M, Chiecchio A, et al. Vascular endothelial growth factor genetic polymorphisms and haplotypes in female patients with bisphosphonate-related osteonecrosis of the jaws. J Oral Pathol Med. 2011; 40: 510-5.

8. Basi DL, Hughes PJ, Thumbigere-Math V, Sabino M, Mariash A, Lunos SA, et al. Matrix metalloproteinase-9 expression in alveolar extraction sockets of Zoledronic acid-treated rats. J Oral Maxillofac Surg. 2011; 69: 2698-707.

9. Manzano-Moreno FJ, Ramos-Torrecillas J, Melguizo-Rodriguez L, Illescas-Montes R, Ruiz C, Garcia-Martinez O. Bisphosphonate Modulation of the Gene Expression of Different Markers Involved in Osteoblast Physiology: Possible Implications in Bisphosphonate-Related Osteonecrosis of the Jaw. International journal of medical sciences. 2018; 15: 359-67.

10. Bagan L, Jimenez Y, Leopoldo M, Rubert A, Bagan J. Serum levels of RANKL and OPG, and the RANKL/OPG ratio in bisphosphonate-related osteonecrosis of the jaw: Are they useful biomarkers for the advanced stages of osteonecrosis? Med Oral Patol Oral Cir Bucal. 2017; 22: e542-e7.

11. Thumbigere-Math V, Michalowicz BS, Hughes PI, Basi DL, Tsai ML, Swenson KK, et al. Serum Markers of Bone Turnover and Angiogenesis in Patients With Bisphosphonate-Related Osteonecrosis of the Jaw After Discontinuation of 
Long-Term Intravenous Bisphosphonate Therapy. J Oral Maxillofac Surg. 2016; 74: 738-46.

12. Kim JW, Cha IH, Kim SJ, Kim MR. Biomarkers for Bisphosphonate-Related Osteonecrosis of the Jaw. Clin Implant Dent Relat Res. 2016; 18: 281-91.

13. Kolokythas A, Karras M, Collins E, Flick W, Miloro M, Adami G. Salivary Biomarkers Associated With Bone Deterioration in Patients With Medication-Related Osteonecrosis of the Jaws. J Oral Maxillofac Surg. 2015; 73: 1741-7.

14. Turchinovich A, Weiz L, Burwinkel B. Extracellular miRNAs: the mystery of their origin and function. Trends Biochem Sci. 2012.

15. Zhou J, Yu L, Gao X, Hu J, Wang J, Dai Z, et al. Plasma microRNA panel to diagnose hepatitis B virus-related hepatocellular carcinoma. J Clin Oncol. 2011; 29: 4781-8.

16. Egea V, Schober A, Weber C. Circulating miRNAs: messengers on the move in cardiovascular disease. Thromb Haemost. 2012; 108: 590-1.

17. Hackl M, Heilmeier U, Weilner S, Grillari J. Circulating microRNAs as novel biomarkers for bone diseases - Complex signatures for multifactorial diseases? Mol Cell Endocrinol. 2016; 432: 83-95.

18. Musolino C, Oteri G, Allegra A, Mania M, D'Ascola A, Avenoso A, et al. Altered microRNA expression profile in the peripheral lymphoid compartment of multiple myeloma patients with bisphosphonate-induced osteonecrosis of the jaw. Ann Hematol. 2018.

19. Howie RN, Borke JL, Kurago Z, Daoudi A, Cray J, Zakhary IE, et al. A Model for Osteonecrosis of the Jaw with Zoledronate Treatment following Repeated Major Trauma. PLoS One. 2015; 10: e0132520.

20. Li H, Yang F, Wang Z, Fu Q, Liang A. MicroRNA-21 promotes osteogenic differentiation by targeting small mothers against decapentaplegic 7 . Molecular medicine reports. 2015; 12: 1561-7.

21. Yang N, Wang G, Hu C, Shi Y, Liao L, Shi S, et al. Tumor necrosis factor alpha suppresses the mesenchymal stem cell osteogenesis promoter miR-21 in estrogen deficiency-induced osteoporosis. J Bone Miner Res. 2013; 28: 559-73.

22. Li C, Li C, Yue J, Huang X, Chen M, Gao J, et al. miR-21 and miR-101 regulate PLAP-1 expression in periodontal ligament cells. Molecular medicine reports. 2012; 5: 1340-6.

23. Pitari MR, Rossi M, Amodio N, Botta C, Morelli E, Federico C, et al. Inhibition of miR-21 restores RANKL/OPG ratio in multiple myeloma-derived bone marrow stromal cells and impairs the resorbing activity of mature osteoclasts. Oncotarget. 2015; 6: 27343-58.

24. Zhou Y, Liu Y, Cheng L. miR-21 expression is related to particle-induced osteolysis pathogenesis. J Orthop Res. 2012; 30: 1837-42.

25. Hu CH, Sui BD, Du FY, Shuai Y, Zheng CX, Zhao P, et al. miR-21 deficiency inhibits osteoclast function and prevents bone loss in mice. Sci Rep. 2017; 7: 43191.

26. Zeng HC, Bae Y, Dawson BC, Chen Y, Bertin T, Munivez E, et al. MicroRNA miR-23a cluster promotes osteocyte differentiation by regulating TGF-beta signalling in osteoblasts. Nat Commun. 2017; 8: 15000 .

27. Guo $Q$, Chen $Y$, Guo L, Jiang T, Lin Z. miR-23a/b regulates the balance between osteoblast and adipocyte differentiation in bone marrow mesenchymal stem cells. Bone research. 2016; 4: 16022.

28. Li T, Li H, Wang Y, Li T, Fan J, Xiao K, et al. microRNA-23a inhibits osteogenic differentiation of human bone marrow-derived mesenchymal stem cells by targeting LRP5. Int J Biochem Cell Biol. 2016; 72: 55-62.

29. Dong Y, Li T, Li Y, Ren S, Fan J, Weng X. MicroRNA-23a-3p inhibitor decreases osteonecrosis incidence in a rat model. Molecular medicine reports. 2017; 16: 9331-6.

30. Sun K, Wang J, Liu F, Ji Z, Guo Z, Zhang C, et al. Ossotide promotes cell differentiation of human osteoblasts from osteogenesis imperfecta patients by up-regulating miR-145. Biomed Pharmacother. 2016; 83: 1105-10.

31. Yu FY, Xie CQ, Sun JT, Peng W, Huang XW. Overexpressed miR-145 inhibits osteoclastogenesis in RANKL-induced bone marrow-derived macrophages and ovariectomized mice by regulation of Smad3. Life Sci. 2018; 202: 11-20.

32. Tian ZJ, Liu BY, Zhang YT, Chen XZ, Qiao GY, Wang S, et al. MiR-145 silencing promotes steroid-induced avascular necrosis of the femoral head repair via upregulating VEGF. Eur Rev Med Pharmacol Sci. 2017; 21: 3763-9. 